\title{
C13C4.5/Spinster, an evolutionarily conserved protein that regulates fertility in C. elegans through a lysosome-mediated lipid metabolism process
}

\author{
Mei Han ${ }^{1,2}$, Hao Chang ${ }^{1,3}$, Peng Zhang ${ }^{1,2}$, Tao Chen ${ }^{4}$, Yanhua Zhao ${ }^{1}$, Yongdeng Zhang ${ }^{1}$, Pingsheng Liu ${ }^{1}$, \\ Tao $\mathrm{Xu}^{1 \bowtie}$, Pingyong $\mathrm{Xu}^{5 \bowtie}$ \\ ${ }^{1}$ National Laboratory of Biomacromolecules, Institute of Biophysics, Chinese Academy of Sciences, Beijing 100101, China \\ 2 University of Chinese Academy of Sciences, Beijing 100049, China \\ ${ }^{3}$ School of Life Sciences, University of Science and Technology of China, Hefei 230027, China \\ ${ }^{4}$ College of Engineering, and Biodynamic Optical Imaging Center (BIOPIC), Peking University, Beijing 100871, China \\ ${ }^{5}$ Key Laboratory of Interdisciplinary Research, Institute of Biophysics, Chinese Academy of Sciences, Beijing 100101, China \\ $\triangle$ Correspondence: pyxu@ibp.ac.cn (P. Xu), xutao@ibp.ac.cn (T. Xu) \\ Received February 17, 2013 Accepted March 27, 2013
}

\begin{abstract}
Lipid droplets, which are conserved across almost all species, are cytoplasmic organelles used to store neutral lipids. Identification of lipid droplet regulators will be conducive to resolving obesity and other fat-associated diseases. In this paper, we selected 11 candidates that might be associated with lipid metabolism in Caenorhabditis elegans. Using a BODIPY 493/503-based flow cytometry screen, 6 negative and 3 positive regulators of fat content were identified. We selected one negative regulator of lipid content, C13C4.5, for future study. C13C4.5 was mainly expressed in the worm intestine. We found that this gene was important for maintaining the metabolism of lipid droplets. Biochemical results revealed that $50 \%$ of triacylglycerol (TAG) was lost in C13C4.5 knockout worms. Stimulated Raman scattering (SRS) signals in C13C4.5 mutants showed only $49.6 \%$ of the fat content in the proximal intestinal region and $86.3 \%$ in the distal intestinal region compared with wild type animals. The mean values of lipid droplet size and intensity in C13C4.5 knockout animals were found to be significantly decreased compared with those in wild type worms. The LMP-1-labeled membrane structures in worm intestines were also enlarged in C13C4.5 mutant animals. Finally, fertility defects were found in C13C4.5(ok2087) mutants. Taken together, these results indicate that C13C4.5 may regulate the fertility of
\end{abstract}

C. elegans by changing the size and fat content of lipid droplets by interfering with lysosomal morphology and function.

KEYWORDS C13C4.5, Spinster, lipid droplet, SRS microscopy, lysosomal enlargement, fertility

\section{INTRODUCTION}

Lipid droplets are found in nearly all types of cells and have been described as intracellular storage organelles for neutral lipids, including triacylglycerols and cholesteryl esters (Murphy, 2001; Beckman, 2006; Martin and Parton, 2006). Lipid droplets were first observed and described by van Leeuwenhoek in the year 1674 using his self-made microscope (Kernohan and Lepherd, 1969). In the 19th century, lipid droplets were visualized in cells by light microscopy (Walther and Farese, 2012). In recent years, lipid droplets were found to be linked to other internal membrane systems such as the endoplasmic reticulum, endosomes, and peroxisomes (Goodman, 2008; Murphy et al., 2009; Zehmer et al., 2009; Walther and Farese, 2012). The pathogenesis of various viruses and bacteria, such the hepatitis $C$ virus, dengue virus and Chlamydia, all involve lipid droplets (Cocchiaro et al., 2008; Samsa et al., 2009; Herker and Ott, 2011). In metazoans, lipid droplets are present in various sizes and numbers in different tissues during different developmental periods. Lipid droplets reflect metabolic status and diet 
conditions. Metabolic diseases such as obesity, diabetes and atherosclerosis are linked to changes in lipid droplet morphology. Thus, research into the mechanisms of lipid droplet regulators will be conducive to disease diagnosis and treatment.

To identify modulators of fat storage, RNAi-based whole genomic screens have been performed in many organisms including Caenorhabditis elegans, Drosophila melanogaster and S2 cells (Ashrafi et al., 2003; Szymanski et al., 2007; Fei et al., 2008; Guo et al., 2008; Pospisilik et al., 2010). C. elegans is an excellent genetic model system with many advantages for studying lipid droplets. First, the whole genomic RNAi feeding library is established and is easily used for specific gene knocking down (Kamath et al., 2003). Second, staining methods containing oil Red O and BODIPY 493/503 dyes have been used and confirmed to quantify fat content in fixed worms (Brooks et al., 2009; O'Rourke et al., 2009; Klapper et al., 2011). Third, the newly developed label-free coherent antiStokes Raman scattering (CARS) microscopy and stimulated Raman scattering (SRS) microscopy were used to directly image lipid droplets in C. elegans (Watts, 2009; Yen et al., 2010; Wang et al., 2011). However, because the RNAi library cannot cover the whole genome and RNAi efficiency varies greatly among different genes, novel genes still need to be further identified, and a detailed analysis of how those candidates regulate lipid droplets is still required.

In this study, we selected 11 candidate genes in C. elegans that we thought might participate in lipid metabolism and examined their regulation of lipid droplets. Using RNAi feeding and a BODIPY 493/503-based flow cytometry screen, 6 negative regulators (C13C4.5, F42H10.6, F13C5.6, Y71H2AM.9, $\mathrm{F} 13 \mathrm{D} 12.9$ and $\mathrm{F} 36 \mathrm{~A} 2.2)$ and 3 positive regulators (F47H4.4, F53C11.3 and ZK550.6) of fat content were identified. One of these regulators, $\mathrm{C} 13 \mathrm{C} 4.5$, had a significant influence on lipid droplets when depleted. C13C4.5 is a homolog of mammalian Spinster that encodes a late endosomal/lysosomal sugar carrier of the major facilitator superfamily (Nakano et al., 2001; Dermaut et al., 2005). In Drosophila spinster mutants, lysosomes were larger than those in wild type flies (Dermaut et al., 2005). In mammalian cells, defects in Spinster lead to an accumulation of enlarged LAMP1-positive structures (Rong et al., 2011). Spinster was also found to take part in autophagic lysosome reformation (Rong et al., 2011). The localization and function of C13C4.5 have not yet been described in C. elegans. In the current study, we found that $\mathrm{C} 13 \mathrm{C} 4.5$ was mainly expressed in the intestine and hypodermis of $C$. elegans (Fig. S1). The deletion of the C13C4.5 gene significantly decreased offspring. We found that the C13C4.5(ok2087) mutant strain contained only $50 \%$ of the TAG level of wild type. Both oil Red O staining and SRS imaging showed that loss of C13C4.5 decreased the size of lipid droplets as well as their fat content. Confocal microscopy showed that the C13C4.5 mutants also exhibited enlarged LMP-1-positive structures in the intestine. Based on these results, we propose that $\mathrm{C} 13 \mathrm{C} 4.5$ regulates the number of offspring in $C$. elegans by changing the size and fat content of lipid droplets through interference with lysosome morphol- ogy and function.

\section{RESULTS}

Screen of 11 candidates associated with fat content in C. elegans

We selected 11 candidate genes in $C$. elegans for further study using protein sequence alignments with Rhodococcus sp. RHA1, which was previously reported to hoard large amounts of triacylglycerols (Table 1) (McLeod et al., 2006). All 11 candidates were highly conserved but poorly analyzed in worms, and none had been reported to have a relationship with fat mass, mainly due to the absence of knockout strains. A novel BODIPY 493/503-based fat staining and flow cytometry protocol has been developed to measure neutral lipid content and body proportion simultaneously (Klapper et al., 2011). C. elegans genes are efficiently inactivated by feeding with RNAi bacteria (Fire et al., 1998; Kamath et al., 2003). Synchronized young adults with inactivated genes were fixed, stained with BODIPY 493/503 and then analyzed using complex object parametric analysis and sorter (COPAS). We selected daf-7(e1372) mutants as the positive control. C. elegans fed with L4440 empty vector-containing bacteria were used to normalize COPAS readout values. The daf-7(e1372) mutants exhibited a $14 \%$ increase, which matched the tendency previously reported (Table 1). RNAi of 6 candidate genes (C13C4.5, F42H10.6, F13C5.6, Y71H2AM.9, F13D12.9 and F36A2.2) decreased fat content by more than $10 \%$. Interestingly, the homolog of F42H10.6, Acot13 (Blast e-value: $6.4 \times 10^{-24}$ ), decreased free fatty acid concentration by $23 \%$ when depleted from mouse liver (Kang et al., 2012). In addition, deletion of another three candidates, F47H4.4, F53C11.3 and ZK550.6, increased neutral lipids by more than $10 \%$ compared with wild type. Among

Table 1. Screen of 11 candidate genes by BODIPY $493 / 503$

\begin{tabular}{llll}
\hline Gene & Locus & $\begin{array}{l}\text { BODIPY } \\
493 / 503 \\
\text { fluores- } \\
\text { cence } \\
\text { intensity }\end{array}$ & $\begin{array}{l}\text { Number of } \\
\text { animals } \\
\text { analyzed with } \\
\text { BODIPY } \\
\text { 493/503 staining }\end{array}$ \\
\hline B0412.2 & daf-7 & 1.1381 & 320 \\
F47H4.4 & fbxa-185 & 1.4655 & 813 \\
ZK593.8 & fic-1 & 0.9665 & 930 \\
C13C4.5 & & 0.8396 & 694 \\
ZK550.6 & & 1.1028 & 856 \\
F53C11.3 & & 1.0869 & 928 \\
Y51A2B.2 & & 1.0164 & 1306 \\
F42H10.6 & & 0.8733 & 896 \\
F13C5.6 & & 0.8703 & 973 \\
Y71H2AM.9 & & 0.8324 & 373 \\
F13D12.9 & & 0.7815 & 869 \\
F36A2.2 & & 0.8513 & 950 \\
\hline
\end{tabular}


A

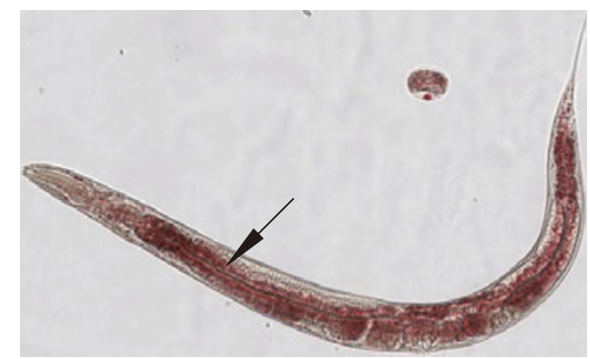

B

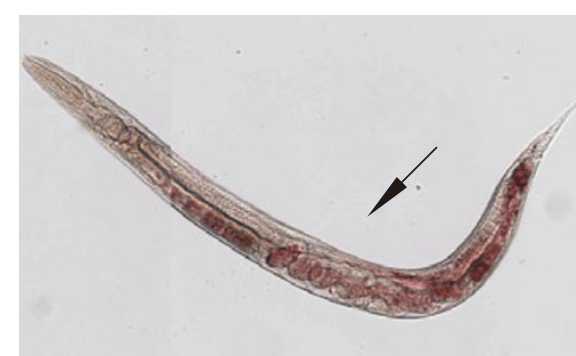

C

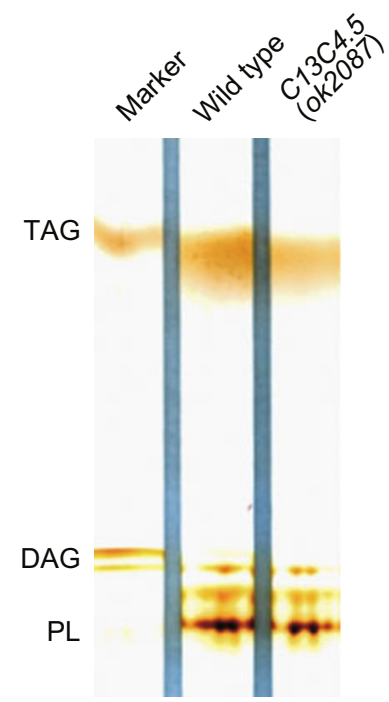

D

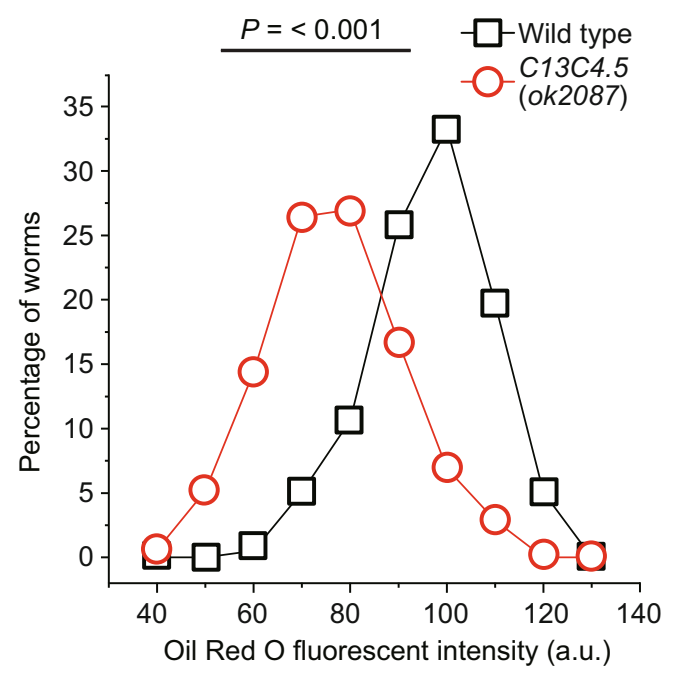

Figure 1. The analysis of a novel fat-mass regulatory gene, C13C4.5, based on oil Red $\mathrm{O}$ staining and thin layer chromatography methods. (A and B) Oil Red O staining images of wild type and C13C4.5(ok2089) (arrow marked proximal intestine region). (C) Quantification of triacylglycerol stores by TLC analysis. Total lipids were extracted from the worms with chloroform:acetone $(1: 1, v / V)$ and separated by thin layer chromatography (TLC) in a hexane:diethyl ether:acetic acid (80:20:1, v/V/V) solvent system. Lane 1, standards. Lane 2, lipids from wild type. Lane 3, lipids from C13C4.5(ok2089). (D) Mean signal distributions of oil Red O-stained wild type and C13C4.5(ok2089).

these candidates, only $\mathrm{C} 13 \mathrm{C} 4.5$ is available as a knockout strain from the Caenorhabditis Genetics Center (CGC). Thus we selected this gene to study lipid storage and its functional relationship with lipid metabolism.

\section{Disruption of C13C4.5 function alters TAG content}

Compared with BODIPY 493/503 staining of lipid droplets, staining with the widely used oil Red $\mathrm{O}$ is a more facile and accurate method to evaluate fat content (Brooks et al., 2009; O'Rourke et al., 2009). In C. elegans, the major fat storage organ is the intestine, and other lipid droplet-containing structures include the hypodermis, gonad, and eggs (O'Rourke et al., 2009; Wang et al., 2011). We calculated the oil Red O signal in the intestine as a proxy for fat mass in worms. Two metabolic mutants, daf-7(e1372) and eat-2(ad465), were used as the positive and negative controls, respectively, to evaluate the stability of the oil Red O signal. Compared with N2, daf$7(e 1372)$ worms exhibited a $24 \%$ increase in oil Red O stain- ing, whereas eat-2(ad465) showed a $34 \%$ decrease, which is consistent with previously published results (Klapper et al., 2011). In the C13C4.5-defective strain C13C4.5(ok2087), we found that the oil Red $\mathrm{O}$ staining signal was only $78 \%$ of that of wild type (Fig. 1). The intestine, which stores major neutral lipids, showed substantially less oil Red $O$ signal in the mutant strain (Fig. 1A, 1B and 1D). This result supports the idea that loss of C13C4.5 downregulated the fat mass in C. elegans.

To further substantiate this conclusion, we extracted total lipids from both the wild type and the C13C4.5(ok2087) strains using chloroform and acetone and then separated different lipid species by thin layer chromatography (TLC). The TLC results showed that the TAG level in wild type was 2-fold higher than that of C13C4.5 mutants (Fig. 1C).

\section{Disruption of C13C4.5 function alters lipid droplet size and content}

SRS microscopy, which enables 3-dimensional lipid droplet 


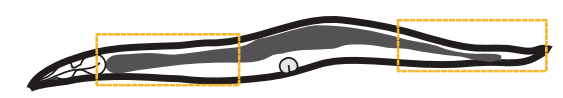

B

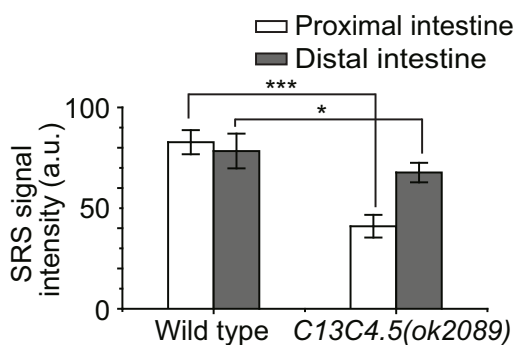

C

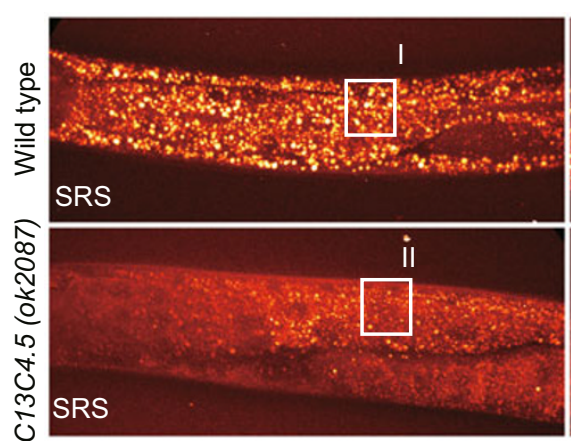

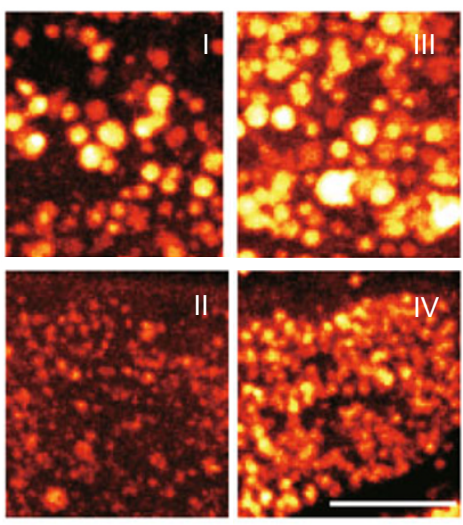

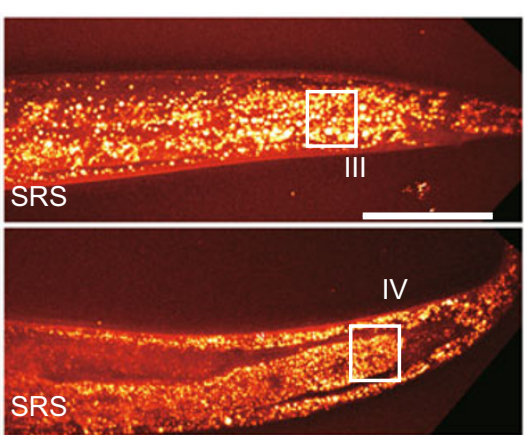

Figure 2. Distribution of fat mass in wild type and C13C4.5 mutants using SRS microscopy. (A) The SRS imaging region in C. elegans. (B) Quantification of lipid content in proximal and distal intestinal regions for wild type and $C 13 C 4.5$ mutants $\left({ }^{* \star *} P<0.001\right.$, ${ }^{*} P<0.05$ ). (C) Representative SRS microscopy images of wild type and C13C4.5 mutants. (D) Amplified photographs of boxes I-IV in C. Scale bars: $50 \mu \mathrm{m}$ in $\mathrm{C}$ and $10 \mu \mathrm{m}$ in $\mathrm{D}$.

visualization with high sensitivity and specificity, was used to analyze single lipid droplets of wild type and C13C4.5(ok2087) worms. Unlike CARS and other indirect dye-staining methods, the SRS signal is linear for lipids without unrelated background, which allows exact determination of the concentration, size, distribution and number of lipid droplets in single worms (Freudiger et al., 2008; Wang et al., 2011). We evaluated the mean intensity of the SRS signal for young adult worm in the proximal intestine and the distal intestine (Fig. 2A). Compared with wild type, the C13C4.5(ok2087) strain exhibited only $49.6 \%$ of the fat content in the proximal intestinal region and $86.3 \%$ in the distal intestinal region (Fig. 2B). Lipid storage in both the intestine and the hypodermis was decreased in C13C4.5 loss-of-function worms (Fig. 2C). We imaged lipid droplets at a higher magnification and found that the size and SRS signal for single lipid droplet decreased significantly in both regions when C13C4.5 was depleted (Fig. 2D).

We next examined the detailed changes in lipid droplets using SRS microscopy (Fig. 3). The average diameter and volume of the lipid droplets in C13C4.5 mutants were approximately $84 \%$ and $70 \%$, respectively, of those in N2 animals (Fig. 3A and 3B). The SRS signal of single lipid droplets was calculated, and the mean value of signal intensity in C13C4.5 mutants was approximately $37 \%$ of that in wild type
(Fig. 3C). Although the SRS signal intensity of single lipid droplets was positively correlated with their size in both wild type and C13C4.5 mutants, the slopes showed distinct values (Fig. 3D). Lipid droplets from 20,000 to 70,000 (SRS signal of lipid droplets by Gaussian fitting) were especially rare in mutants. Even for droplets with similar diameters larger than 1 $\mu \mathrm{m}$, much more fat was detected in wild type lipid droplets than in those of the mutants (Figs. 2D and 3D). This result suggests that the $C 13 C 4.5$ gene is an important positive regulator for lipid biosynthesis and storage. However, the total number of lipid droplets in the intestine did not statistically differ between wild type and C13C4.5 mutants ( $P$ value $=0.263$ ), which implies that the $C 13 C 4.5$ gene had no influence on the density of the lipid droplets. Interesting, although most lipid droplets from the mutant worms are much smaller than those of wild type, one or more abnormal large lipid droplets with a size of approximately $4 \mu \mathrm{m}$ were occasionally found in the tail hypodermis (Fig. S2). We hypothesize that those large lipid droplets might be a compensatory mechanism of lipid decrease in C13C4.5 mutants.

To confirm SRS results, oil Red O stained wild type and C13C4.5 mutants were re-examined using a upside dissection microscopy with a higher amplification objective. In Figure S3, compared with wild type, C13C4.5-defective strain exhibits 
A

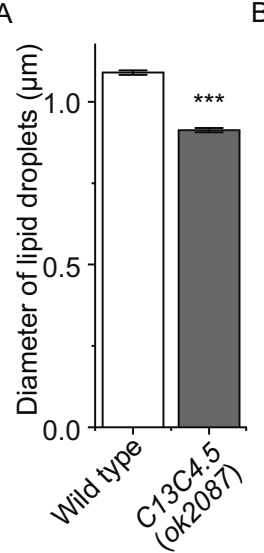

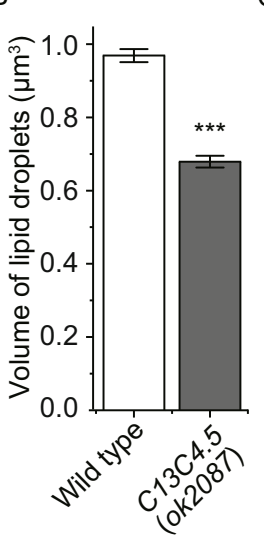

C

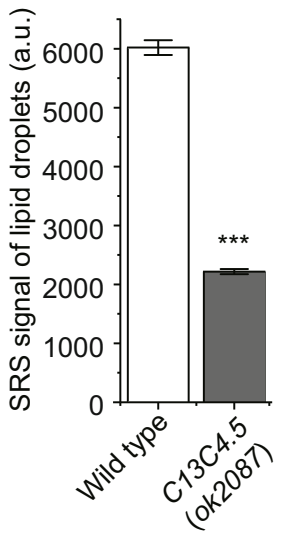

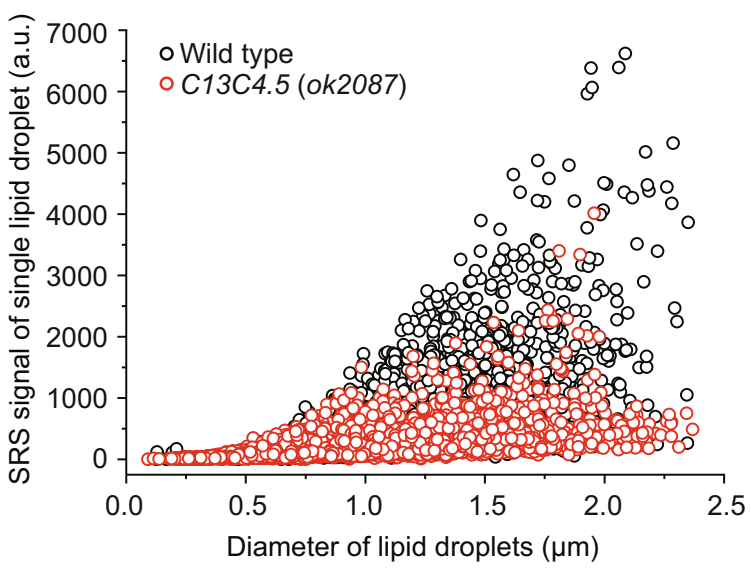

Figure 3. Lipid droplet size and SRS signal intensity in C13C4.5 mutants. (A-C) Mean diameter, volume and signal intensity of SRS signal-positive lipid droplets in wild type and C13C4.5 mutants. (D) The size of each lipid droplet was positively correlated with SRS signal intensity in the plotted distribution.

smaller lipid droplets in the proximal intestine, which matches the result of SRS microscopy very well. For further demonstration, we injected the construct PC13C4.5::C13C4.5::GFP and obtained the stable strain. In this C13C4.5-rescue strain, the phenotype of lipid droplets is successfully rescued which reflects that $\mathrm{C} 13 \mathrm{C} 4.5$ is one of key elements to mediate the lipid droplet size and content (Fig. S3).

The C13C4.5 mutant exhibited abnormal lysosomal/late endosomal structures but normal early endosomes

To evaluate the effects of $\mathrm{C} 13 \mathrm{C} 4.5$ on the components of the endomembrane system such as early endosomes, late endosomes and lysosomes, the C13C4.5 mutant gene was crossed into each of the mCherry::RAB-5, mCherry::RAB-7 and LMP-1::GFP integrated strains. We then examined these strains by confocal microscopy. The localization and distribution of RAB-5-labeled early endosomes were normal in the C13C4.5 mutant strain compared with wild type, although
RAB-7-marked late endosomes exhibited a slightly abnormal morphology in some mutant worms (Fig. S4). Significantly, compared with wild type worms, many enlarged lysosomal structures labeled by LMP-1 were observed in the C13C4.5 mutants (Fig. 4A and 4B). After $6 \mathrm{~h}$ of starvation, the lysosomal size was slightly decreased, but the abnormal lysosomal morphology was still seen in C13C4.5 mutants compared with wild type worms (Fig. 4C and 4D). Under normal conditions, the size of enlarged lysosomes after C13C4.5 deletion was approximately 3 - to 5 -fold larger than that of normal worms (Fig. 4E and 4F). We propose that the abnormal size and morphology of the lysosomes induced by $\mathrm{C} 13 \mathrm{C} 4.5$ deletion may be the main reason for the defect in intestinal cell fat storage.

\section{Fertility defects in C13C4.5(ok2087) mutants}

We next examined the biological effects of defective fat storage in intestinal cells by analyzing the phenotypes of $C 13 C 4.5$ mutants. We compared worm length, width and length/width ratio and found no significant differences between wild type and C13C4.5(ok2087) animals (Fig. S5). There was also no difference in behaviors such as pumping and defecation in the mutant worms compared with wild type (Fig. 5A-C). These results suggest that the $C 13 C 4.5$ gene may not influence the growth and development of $C$. elegans. However, when we counted and compared the average live progeny produced per worm, there was a significant difference between wild type and C13C4.5(ok2087) mutants, suggesting that $C 13 C 4.5$ plays an important role in fertility (Fig. 5D).

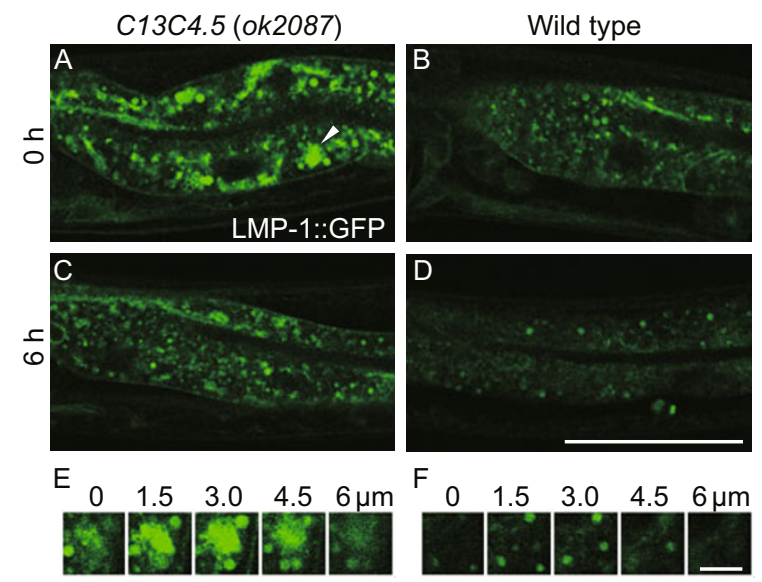

Figure 4. The LMP-1-positive membrane system in C13C4.5 mutants. (A and B) Representative photographs of LMP-1-positive structures in C13C4.5 mutants and wild type intestines without starvation ( $n=25)$. (C and D) Representative photographs of LMP-1-positive structures in C13C4.5 mutants and wild type intestines after $6 \mathrm{~h}$ of starvation $(n=25)$. (E) Representative z-axis slices of abnormal lysosomes in C13C4.5 mutants (arrowhead in A) (F) Representative z-axis slices of normal lysosomes in wild type worms. Scale bars: $50 \mu \mathrm{m}$ in A, B, C and D; $5 \mu \mathrm{m}$ in $\mathrm{E}$ and $\mathrm{F}$. 


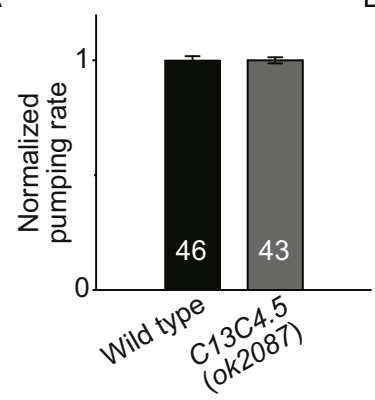

C

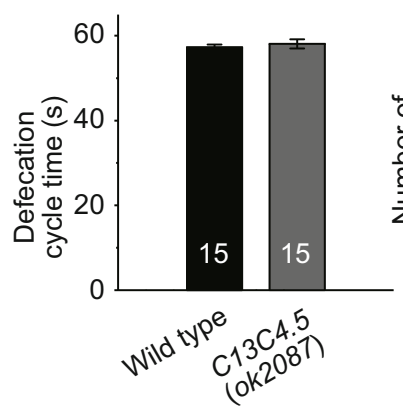

D
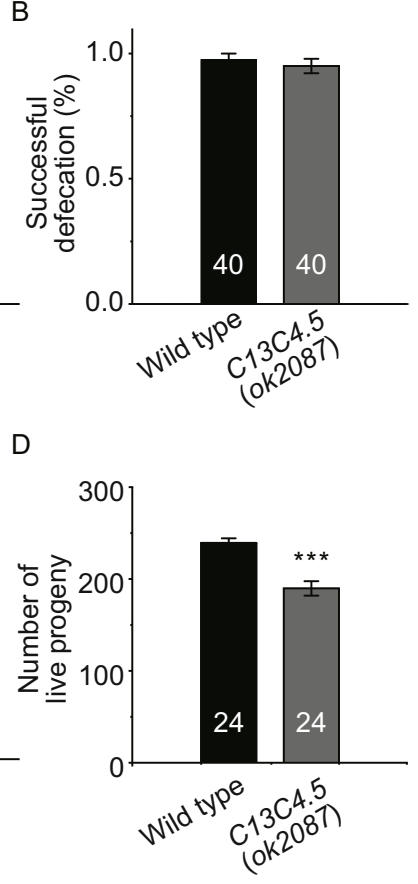

Figure 5. Phenotypic characterization of pumping, defecation and fertility in C13C4.5 mutants. (A-C) Normalized pumping rate, successful defecation ratio and defecation cycle time were determined under the same conditions and showed no statistically significance differences for wild type and C13C4.5 mutants. (D) Average live progeny produced per adult worm of wild type and C13C4.5 mutants over 4 days (*** $P<0.001)$.

\section{DISCUSSION}

The C13C4.5 gene in C. elegans is one of three homologs of Spinster, which encodes a sugar transporter in the major facilitator superfamily. It has been reported that, in mammalian cells, Spinster is localized to the membrane of late endosomes/lysosomes, and Spinster has been shown to generate lysosomal abnormalities (Rong et al., 2011). In Drosophila Spinster mutants, phenotypes such as defects in endosomalto-lysosomal trafficking, lysosomal enlargement and carbohydrate accumulation have been observed (Nakano et al., 2001; Dermaut et al., 2005). The localization and function of C13C4.5 have not been described in C. elegans. In the current study, we found that $\mathrm{C} 13 \mathrm{C} 4.5$ was mainly expressed in the intestine and the hypodermis of C. elegans (Fig. S1). The C13C4.5 mutant exhibited enlarged lysosomal structures, which were similar to those observed in Drosophila and mammalian cells. Most importantly, we found that $C 13 C 4.5$ regulates the size of lipid droplets and that deletion of this gene significantly decreased their fat content and triacylglycerol levels compared with lipid droplets in wild type animals. It was reported that the mammalian homolog, Spinster, regulates autophagy and lysosome reformation in NRK cells. Most recently, several autophagy genes were found to be required for lipid remodeling in C. elegans (Lapierre et al., 2013). It is likely that C13C4.5 may also regulate autophagy by interfering with the morphology and function of lysosomes and subsequent fat storage and lipid metabolism. However, other possibilities, including direct association of lysosomes with lipid droplets, may also exist, as lysosomes function during many biological processes, and any process such as autophagy that interferes with lysosomes may regulate fat storage and lipid droplets.

The gene $C 13 C 4.5$ is highly conserved from bacteria to humans. The phenotype of a Spinster mutant in animals was described in Drosophila, where it was shown to regulate age-dependent synaptic dysfunction and neuronal degeneration. Here, we report a second physiological role of this gene C13C4.5 in C. elegans. We found this gene functions in the fertility of worms. In previous report, some fat associated genes such as fat- 6 and fat-7 are crucial for maintenance of optimal fertility, might by keeping oleic (18:1D9) and linoleic acids (18:2) (Brock et al., 2007). So we guess that the fertility defect in C13C4.5 mutant worms may be related to the decrease in fat storage and lipid droplets in the intestine (Figs. 1-3). Taken together, these results suggest that $\mathrm{C} 13 \mathrm{C} 4.5$ may function in the fertility of $C$. elegans by regulating fat content and lipid droplets in the intestine, most likely by changing the morphology and function of lysosomes and related autophagy processes.

\section{MATERIALS AND METHODS}

\section{C. elegans strains}

Strain maintenance and genetic manipulation were performed as described previously (Brenner, 1974). Animals were grown at $20{ }^{\circ} \mathrm{C}$ on agar nematode growth media seeded with RNAi feeding bacteria or Escherichia coli strain OP50. The wild type strain was N2 Bristol. RB1678: C13C4.5(ok2087) V was outcrossed four times against N2. C13C4.5(ok2087) V is a C13C4.5-defective strain with a $951 \mathrm{bp}$ deletion in the genome. In this strain, 4-7 exons have been deleted and the last 8th exon has been disrupted by frame-shift mutation. The C13C4.5 rescue strain is obtained by injecting the construct PC13C4.5:: C13C4.5::GFP in C13C4.5(ok2087) mutants.

Other strains used include: KP3948 eri-1(mg366) IV; lin15B(n744) X, CB1372 daf-7(e1372) III, DA465 eat-2(ad465) II, Is[Pges-1::mCherry::RAB-5], Is[Pges-1::mCherry::RAB-7], Is[PImp1::LMP-1::GFP], C13C4.5(ok2087); Is[Pges-1::mCherry::RAB-5], C13C4.5(ok2087); Is[Pges-1::mCherry::RAB-7], and C13C4.5 (ok2087); Is[PImp-1::LMP-1::GFP].

\section{Reagents}

BODIPY 493/503 was purchased from Invitrogen and diluted in DMSO at a concentration of $1 \mathrm{mg} / \mathrm{mL}$ as the stock buffer. $2 \times$ MRWB buffer contained $160 \mathrm{mmol} / \mathrm{L} \mathrm{KCl}, 40 \mathrm{mmol} / \mathrm{L} \mathrm{NaCl}, 14 \mathrm{mmol} / \mathrm{L} \mathrm{Na}{ }_{2}$ EGTA, $1 \mathrm{mmol} / \mathrm{L}$ spermidine- $\mathrm{HCl}, 0.4 \mathrm{mmol} / \mathrm{L}$ spermine, $30 \mathrm{mmol} / \mathrm{L}$ Na-PIPES, $0.2 \% \beta$-mercaptoethanol $(\mathrm{pH}=7.4)$. Oil Red $\mathrm{O}$ working buffer was prepared as follows: oil Red $\mathrm{O}$ was first diluted into isopropanol at a concretion of $0.5 \mathrm{~g} / \mathrm{mL}$ and rocked for several days, then diluted to $60 \%$ with water and filtered with a $0.22 \mu \mathrm{m}$ filter.

\section{BODIPY 493/503 staining}

Worms at the stage of day one were harvested in M9 buffer, incubated 
in 3\% paraformaldehyde solution for $15 \mathrm{~min}$ and treated with 3 freeze/ thaw cycles in liquid nitrogen. The buffer was exchanged to M9 three times. Worms were then settled and resuspended in $1 \mu \mathrm{g} / \mathrm{mL}$ BODIPY 493/503 (Invitrogen) in M9 buffer for $1 \mathrm{~h}$ at room temperature. Animals were then washed three times using M9 buffer.

\section{RNAi screen of candidate genes using COPAS}

The RNAi feeding bacteria used were from either Ahringer Library (Kamath et al., 2003) or Vidal Unique Library (Rual et al., 2004; Kim et al., 2005). Feeding bacteria of the 11 candidate genes were picked and cultured at $37^{\circ} \mathrm{C}$ overnight. RNAi feeding bacteria were then seeded on NGM plates containing $5 \mathrm{mmol} / \mathrm{L}$ IPTG. After $24 \mathrm{~h}$, synchronized animals were plated on these induced NGM plates. Progeny were harvested with M9 buffer after 6 days and used immediately for BODIPY $493 / 503$ staining. The stained population was detected and analyzed using a complex object parametric analysis and sorter (COPAS, Union Biometrica) instrument. The COPAS instrument analyzes objects using the physical parameters of object length, optical density, and the intensity of fluorescent markers. Stained animals were placed in 96-well plates and analyzed by COPAS. Quantification data of mixed-stage animals were separated by variant COPAS parameters setting. Adult stage animals were chosen for fluorescent signal quantitative analysis.

\section{Oil Red O staining of fixed nematodes}

Approximately 200-300 day one adult worms were washed with M9 buffer. Worms were then settled and washed three times with M9 buffer. To permeabilize the cuticle, worms were soaked in $2 \%$ paraformaldehyde (PFA) containing MRWB buffer and gently rocked for $60 \mathrm{~min}$. After washing with PBS to remove the PFA, animals were placed into $60 \%$ isopropanol for $15 \mathrm{~min}$ for dehydration. Worms were then settled, resuspended in oil Red O working buffer and incubated overnight with rocking. The dye was removed the following day and PBS with $0.01 \%$ Triton X-100 was added.

\section{Lipid extraction and thin layer chromatography (TLC)}

Lipids were extracted from 4000 L4 worms for TLC analysis using chloroform:acetone $(1: 1, v / v)$. The solvent was removed using N2 gas, and the lipids were dissolved in chloroform and separated on TLC plates in hexane:diethyl ether:acetic acid (80:20:1, v/v) for $40 \mathrm{~min}$. Lipids were visualized by iodine vapor and semiquantified by densitometric scanning (NIH Image J software).

\section{SRS microscopy assembly}

A pump laser integrated Optical Parametric Oscillator (OPO), also known as a one box OPO (APE, Berlin, Germany) served as the light source. It provided a synchronized and spatially overlapped pump and Stokes pulse trains at a repetition rate of $76 \mathrm{MHz}$. The pump beam was continuously tunable from $720-990 \mathrm{~nm}$; however, the Stokes beam was fixed at $1064 \mathrm{~nm}$. The Stokes beam was modulated by an electrooptic modulator (EO-AM-NR-C2, Thorlabs, USA) at a frequency of 9.81 MHz. The collinearly overlapped beams were directed into an inverted multi-photon microscope (IX81/FV1000, Olympus, Japan). Lasers were focused on the sample by a $60 \mathrm{X}$ water immersion objective (UPLSAPO 60XW, Olympus, Japan) and scanned to acquire images. The transmission light was collected by a water immersion condenser
(N.A. 0.9, Olympus, Japan) and then filtered by a bandpass filter (890/220 m, Chroma, USA) to remove the Stokes beam. The pump beam was detected by a large area photodiode (FDS1010, Thorlabs, USA). The voltage signal generated by the photodiode was sent into a home-built lock-in amplifier (designed by Dr. Brian Saar). The demodulated signal was fed back into the microscope software (FV10-ASW, Olympus, Japan) for image reconstruction.

\section{Image acquisition}

Prepared C. elegans samples were scanned by the SRS system described above. The frequency difference of the pump and Stokes beams were set at $2845 \mathrm{~cm}^{-1}$, which is the frequency of symmetric vibration mode of $\mathrm{a} \mathrm{CH}_{2}$ bond, indicating the lipid content in the sample. To obtain complete information about the lipid distribution in the worm intestines, z-stacks with a resolution of $1.5 \mu \mathrm{m}$ were acquired for all samples. All parameters were maintained through image acquisition for both samples.

\section{Confocal microscopy imaging}

As described previously, we used an FV500 laser scanning confocal microscope (Olympus) to obtain images of worms (Chang et al., 2012; Zhang et al., 2012). All the figures were produced using a $60 \times 1.4 \mathrm{NA}$ objective. The maximum power near the rear pupil of the objective was $0.24 \mathrm{~mW}$ for the $488 \mathrm{~nm}$ laser and $0.08 \mathrm{~mW}$ for the $561 \mathrm{~nm}$ laser. Images were quantified and analyzed using an Olympus FluoView viewer (Japan) and Image $\mathrm{J}$.

\section{Behavioral assays}

All assays were performed on young adult worms. Worms were cultured on NEM plates seeded with E. coli OP50 at $20^{\circ} \mathrm{C}$. Young adult progeny were transferred to new NGM plates with freshly seeded E. coli OP50 before assays. 5-15 young adult worms were tested for each trial; at least three independent experiments were performed. For the feeding assay, pumping rate was calculated by recording the time required to complete 20 pumps. For the defecation assay, we tested the percentage of successful defecations and the defecation cycle time. Failed defecation was determined to consist of a contraction of the posterior body muscles and a contraction of the anterior body muscles without expulsion. Defecation cycle time was measured by recording the time from one contraction of the posterior body muscles to the next. For the fertility assay, single L4 hermaphrodites of each genotype were picked to a NGM plate seeded with E. coli OP50. Worms were transferred to a fresh plate every $24 \mathrm{~h}$ for 4 days. F1 progeny were counted 2 days after adult worms were removed. Eight worms were measured for each assay per individual genotype; three independent experiments were performed.

\section{ACKNOWLEDGEMENTS}

We gratefully acknowledge support from the National Basic Research Program (973 Program) (Nos. 2013CB910103 and 2010CB912303), the National Natural Science Foundation of China (Grant Nos. 31170818 and 31270910), and the project from the Chinese Academy of Sciences (KSCX2-EW-Q-11). We thank Xiaochen Wang and Hong Zhang at the Institute of Biophysics for sharing protein-labeled worm strains. We thank the CGC for provided mutant strains. We thank Xu- 
dong Zhao, Su Liu and Junfeng Hao of the Institute of Biophysics for technical support.

\section{COMPLIANCE WITH ETHICS GUIDELINES}

Mei Han, Hao Chang, Peng Zhang, Tao Chen, Yanhua Zhao, Yongdeng Zhang, Pingsheng Liu, Tao Xu, and Pingyong $\mathrm{Xu}$ declare that they have no conflict of interest.

All institutional and national guidelines for the care and use of laboratory animals were followed.

\section{REFERENCES}

Ashrafi, K., Chang, F.Y., Watts, J.L., Fraser, A.G., Kamath, R.S., Ahringer, J., and Ruvkun, G. (2003). Genome-wide RNAi analysis of Caenorhabditis elegans fat regulatory genes. Nature 421, 268-272.

Beckman, M. (2006). Cell biology. Great balls of fat. Science 311, 1232-1234.

Brenner, S. (1974). The genetics of Caenorhabditis elegans. Genetics 77, 71-94.

Brock, T.J., Browse, J., and Watts, J.L. (2007). Fatty acid desaturation and the regulation of adiposity in Caenorhabditis elegans. Genetics 176, 865-875.

Brooks, K.K., Liang, B., and Watts, J.L. (2009). The influence of bacterial diet on fat storage in C. elegans. PloS One 4, e7545.

Chang, H., Zhang, M., Ji, W., Chen, J., Zhang, Y., Liu, B., Lu, J., Zhang, J., Xu, P., and Xu, T. (2012). A unique series of reversibly switchable fluorescent proteins with beneficial properties for various applications. Proc Natl Acad Sci U S A 109, 4455-4460.

Cocchiaro, J.L., Kumar, Y., Fischer, E.R., Hackstadt, T., and Valdivia, R.H. (2008). Cytoplasmic lipid droplets are translocated into the lumen of the Chlamydia trachomatis parasitophorous vacuole. Proc Natl Acad Sci U S A 105, 9379-9384.

Dermaut, B., Norga, K.K., Kania, A., Verstreken, P., Pan, H., Zhou, Y., Callaerts, P., and Bellen, H.J. (2005). Aberrant lysosomal carbohydrate storage accompanies endocytic defects and neurodegeneration in Drosophila benchwarmer. J Cell Biol 170, 127-139.

Fei, W., Shui, G., Gaeta, B., Du, X., Kuerschner, L., Li, P., Brown, A.J., Wenk, M.R., Parton, R.G., and Yang, H. (2008). Fld1p, a functional homologue of human seipin, regulates the size of lipid droplets in yeast. J Cell Biol 180, 473-482.

Fire, A., Xu, S., Montgomery, M.K., Kostas, S.A., Driver, S.E., and Mello, C.C. (1998). Potent and specific genetic interference by doublestranded RNA in Caenorhabditis elegans. Nature 391, 806-811.

Freudiger, C.W., Min, W., Saar, B.G., Lu, S., Holtom, G.R., He, C., Tsai, J.C., Kang, J.X., and Xie, X.S. (2008). Label-free biomedical imaging with high sensitivity by stimulated Raman scattering microscopy. Science 322, 1857-1861.

Goodman, J.M. (2008). The gregarious lipid droplet. J Biol Chem 283, 28005-28009.

Guo, Y., Walther, T.C., Rao, M., Stuurman, N., Goshima, G., Terayama, K., Wong, J.S., Vale, R.D., Walter, P., and Farese, R.V. (2008). Functional genomic screen reveals genes involved in lipid-droplet formation and utilization. Nature 453, 657-661.

Herker, E., and Ott, M. (2011). Unique ties between hepatitis C virus replication and intracellular lipids. Trends Endocrin Met 22, 241-248.

Kamath, R.S., Fraser, A.G., Dong, Y., Poulin, G., Durbin, R., Gotta, M., Kanapin, A., Le Bot, N., Moreno, S., Sohrmann, M., et al. (2003).
Systematic functional analysis of the Caenorhabditis elegans genome using RNAi. Nature 421, 231-237.

Kang, H.W., Niepel, M.W., Han, S., Kawano, Y., and Cohen, D.E. (2012). Thioesterase superfamily member $2 /$ acyl-CoA thioesterase 13 (Them2/Acot13) regulates hepatic lipid and glucose metabolism. FASEB J 26, 2209-2221.

Kernohan, E.A., and Lepherd, E.E. (1969). Size distribution of fat globules in cows milk during milking, measured with a coulter counter. $J$ Dairy Res 36, 177.

Kim, J.K., Gabel, H.W., Kamath, R.S., Tewari, M., Pasquinelli, A., Rual, J.F., Kennedy, S., Dybbs, M., Bertin, N., Kaplan, J.M., et al. (2005). Functional genomic analysis of RNA interference in C. elegans. Science 308, 1164-1167.

Klapper, M., Ehmke, M., Palgunow, D., Bohme, M., Matthaus, C., Bergner, G., Dietzek, B., Popp, J., and Doring, F. (2011). Fluorescence-based fixative and vital staining of lipid droplets in Caenorhabditis elegans reveal fat stores using microscopy and flow cytometry approaches. J Lipid Res 52, 1281-1293.

Lapierre, L.R., Silvestrini, M.J., Nunez, L., Ames, K., Wong, S., Le, T.T., Hansen, M., and Melendez, A. (2013). Autophagy genes are required for normal lipid levels in C. elegans. Autophagy 9.

Martin, S., and Parton, R.G. (2006). Lipid droplets: a unified view of a dynamic organelle. Nature reviews. Mol Cell Biol 7, 373-378.

McLeod, M.P., Warren, R.L., Hsiao, W.W., Araki, N., Myhre, M., Fernandes, C., Miyazawa, D., Wong, W., Lillquist, A.L., Wang, D., et al. (2006). The complete genome of Rhodococcus sp. RHA1 provides insights into a catabolic powerhouse. Proc Natl Acad Sci U S A 103, 15582-15587.

Murphy, D.J. (2001). The biogenesis and functions of lipid bodies in animals, plants and microorganisms. Prog Lipid Res 40, 325-438.

Murphy, S., Martin, S., and Parton, R.G. (2009). Lipid droplet-organelle interactions; sharing the fats. Biochim Biophys Acta 1791, 441-447.

Nakano, Y., Fujitani, K., Kurihara, J., Ragan, J., Usui-Aoki, K., Shimoda, L., Lukacsovich, T., Suzuki, K., Sezaki, M., Sano, Y., et al. (2001). Mutations in the novel membrane protein spinster interfere with programmed cell death and cause neural degeneration in Drosophila melanogaster. Mol Cell Biol 21, 3775-3788.

O'Rourke, E.J., Soukas, A.A., Carr, C.E., and Ruvkun, G. (2009). C. elegans major fats are stored in vesicles distinct from lysosomerelated organelles. Cell Metab 10, 430-435.

Pospisilik, J.A., Schramek, D., Schnidar, H., Cronin, S.J., Nehme, N.T., Zhang, X., Knauf, C., Cani, P.D., Aumayr, K., Todoric, J., et al. (2010). Drosophila genome-wide obesity screen reveals hedgehog as a determinant of brown versus white adipose cell fate. Cell 140, 148-160.

Rong, Y., McPhee, C.K., Deng, S., Huang, L., Chen, L., Liu, M., Tracy, K., Baehrecke, E.H., Yu, L., and Lenardo, M.J. (2011). Spinster is required for autophagic lysosome reformation and $\mathrm{mTOR}$ reactivation following starvation. Proc Natl Acad Sci U S A 108, 7826-7831.

Rual, J.F., Ceron, J., Koreth, J., Hao, T., Nicot, A.S., Hirozane-Kishikawa, T., Vandenhaute, J., Orkin, S.H., Hill, D.E., van den Heuvel, S., et al. (2004). Toward improving Caenorhabditis elegans phenome mapping with an ORFeome-based RNAi library. Genome Res 14, 2162-2168.

Samsa, M.M., Mondotte, J.A., Iglesias, N.G., Assuncao-Miranda, I., Barbosa-Lima, G., Da Poian, A.T., Bozza, P.T., and Gamarnik, A.V. (2009). Dengue virus capsid protein usurps lipid droplets for viral 
particle formation. PLoS Pathog 5, e1000632.

Szymanski, K.M., Binns, D., Bartz, R., Grishin, N.V., Li, W.P., Agarwal, A.K., Garg, A., Anderson, R.G., and Goodman, J.M. (2007). The lipodystrophy protein seipin is found at endoplasmic reticulum lipid droplet junctions and is important for droplet morphology. Proc Natl Acad Sci U S A 104, 20890-20895.

Walther, T.C., and Farese, R.V., Jr. (2012). Lipid droplets and cellular lipid metabolism. Ann Rev Biochem 81, 687-714.

Wang, M.C., Min, W., Freudiger, C.W., Ruvkun, G., and Xie, X.S. (2011). RNAi screening for fat regulatory genes with SRS microscopy. Nat Methods 8, 135-138.

Watts, J.L. (2009). Fat synthesis and adiposity regulation in Caeno- rhabditis elegans. Trends Endocrinol Metab 20, 58-65.

Yen, K., Le, T.T., Bansal, A., Narasimhan, S.D., Cheng, J.X., and Tissenbaum, H.A. (2010). A comparative study of fat storage quantitation in nematode Caenorhabditis elegans using label and label-free methods. PloS One 5.

Zehmer, J.K., Huang, Y., Peng, G., Pu, J., Anderson, R.G., and Liu, P. (2009). A role for lipid droplets in inter-membrane lipid traffic. Proteomics 9, 914-921.

Zhang, M., Chang, H., Zhang, Y., Yu, J., Wu, L., Ji, W., Chen, J., Liu, B., Lu, J., Liu, Y., et al. (2012). Rational design of true monomeric and bright photoactivatable fluorescent proteins. Nat Methods 9, 727-729. 